\title{
Novel uterine lavage system for recovery of human embryos fertilized and matured in vivo
}

This article was published in the following Dove Press journal:

Medical Devices: Evidence and Research

\author{
Alexander Nadal' \\ Sam Najmabadi ${ }^{2,3}$ \\ Bruce Addis' \\ John E Buster ${ }^{1,4}$ \\ 'Department of Research and \\ Development, Previvo Genetics, San \\ Carlos, CA 94070, USA; ${ }^{2}$ Division of \\ Reproductive Endocrinology and \\ Infertility, Punta Mita Hospital, Punta \\ Mita, Nayarit 63734, Mexico; ${ }^{3}$ Division of \\ Reproductive Endocrinology and \\ Infertility, Center for Reproductive \\ Health and Infertility, Beverly Hills, CA \\ 902II, USA; ${ }^{4}$ Division of Reproductive \\ Endocrinology and Infertility, Warren \\ Alpert Medical School of Brown \\ University, Providence, RI 02903, USA
}

Purpose: In this paper we describe a novel uterine lavage system for the recovery of in vivo preimplantation embryos. Currently, no other method exists to retrieve preimplantation embryos except for in vitro fertilization (IVF).

Methods: A single center, prospective feasibility study was conducted to test a novel uterine lavage system for the recovery of in vivo preimplantation embryos in egg donors and patients seeking pregnancy. Subjects were placed on controlled ovarian hyperstimulation followed by intrauterine insemination (IUI) and uterine lavage performed approximately 4-6 days after IUI. Subjects were followed up for 30 days after the procedure to monitor for safety events.

Results: A total of 134 uterine lavage cycles were performed on 81 subjects (average: 1.7 cycles/subject). Ova (oocytes or embryos) were collected in 53\% (71/134) of the cycles with steady improvement of recovery efficiency over the course of the study, and embryos collected in $42 \%(56 / 134)$ of cycles. Embryos of many stages were collected, but $71 \%$ (96/136) of embryos collected were blastocyst stage embryos which are at the most advanced stage of embryogenesis. Embryos recovered were of good quality based on blastocyst gradings in which $74 \%$ (70/95, 1 blastocyst not graded) of the blastocysts were good quality as determined by the Gardner Scale of Morphology. The procedure was well tolerated with minor side effects. In $8 \%$ of cycles a positive hCG was observed after the lavage indicating some embryos were not recovered by the lavage system.

Conclusion: Through this work the system has been shown to recover embryos from the uterus in a safe and effective manner, thus opening the possibility that uterine lavage may serve as an alternative to IVF where patient indications allow.

Keywords: in vivo embryo, uterine lavage, IVF, assisted reproductive technology, blastocyst

\section{Introduction}

In this report, we describe a novel uterine lavage system for access to and recovery of in vivo fertilized and matured preimplantation human embryos. Predictable, reliable and safe access to healthy in vivo embryos will facilitate the analysis of genetic disorders within individual embryos before implantation in the mother's uterus. Currently, no other method exists to retrieve in vivo preimplantation human embryos. An established alternative, in vitro fertilization (IVF) where fertilization and culture take place in an artificial laboratory (in vitro) environment outside the body, is established as a complex alternative technology indicated for producing pregnancy in women who are infertile. We believe it likely that fertile, childbearing age, healthy women wanting to become pregnant but not needing a fertility
Correspondence: Alexander Nadal

Department of Research and

Development, Previvo Genetics, Inc, 1599

Industrial Rd, San Carlos, CA 94070, USA

Tel +I 3234206343

Email anadal@previvo.com 
treatment desire to know about their future pregnancies. Uterine lavage can provide the same access to embryonic information which IVF provides without the documented risks associated with IVF.

\section{Materials and methods Device overview}

The lavage system is subdivided into two parts: The controller \& the catheter.

The fluid handling controller is a custom designed reusable component with a pump, vacuum, and a series of pre-loaded algorithms for managing the operation of fluid flow. The controller enables the lavage procedure to be a repeatable and simple process and creates flow patterns that would not be possible with manually operated fluid delivery devices. The controller settings are programmed to create a pattern of high volume \& low volume flow $(1-5 \mathrm{~mL} / \mathrm{s})$ that is delivered into the uterus in a series of consistent pulses of about $0.5-4 \mathrm{~s} /$ pulse. The controller maintains the free stream velocity such that the stream is not sufficient to cause tissue injury or dislodge an implanted embryo.
The catheter (Figure 1) portion connects to the controller through the delivery and recovery fluid lines. The catheter is single use, disposable and custom designed to provide a stable platform for handling during operation. The catheter contains a dual co-axial lumen with the inner lumen functioning as the fluid delivery line, and the outer lumen being the fluid collection line. The co-axial bifurcation is accomplished via a manifold. The manifold consists of seven essential parts which create a fluid-tight coupling to separate the delivery and collection flow.

The catheter guide tube is adjustable \pm 45 degrees to accommodate for variable pelvic anatomies. The formable guide is a critical feature providing flexibility for use and helps the operator access the cervix. It is made of heavy-walled 304-V malleable stainless steel. The length of the tube has chord cut laser fenestrations which are symmetrically placed about the axis of the guide tube.

The patient contacting component of the catheter is the catheter tip which has a prefixed length of approximately $6 \mathrm{~cm}$, and $3.8 \mathrm{~mm}$ diameter; it is adjustable to shallow uterine depths with the use of a simple accessory. The tip is made of compliant polymer materials which are

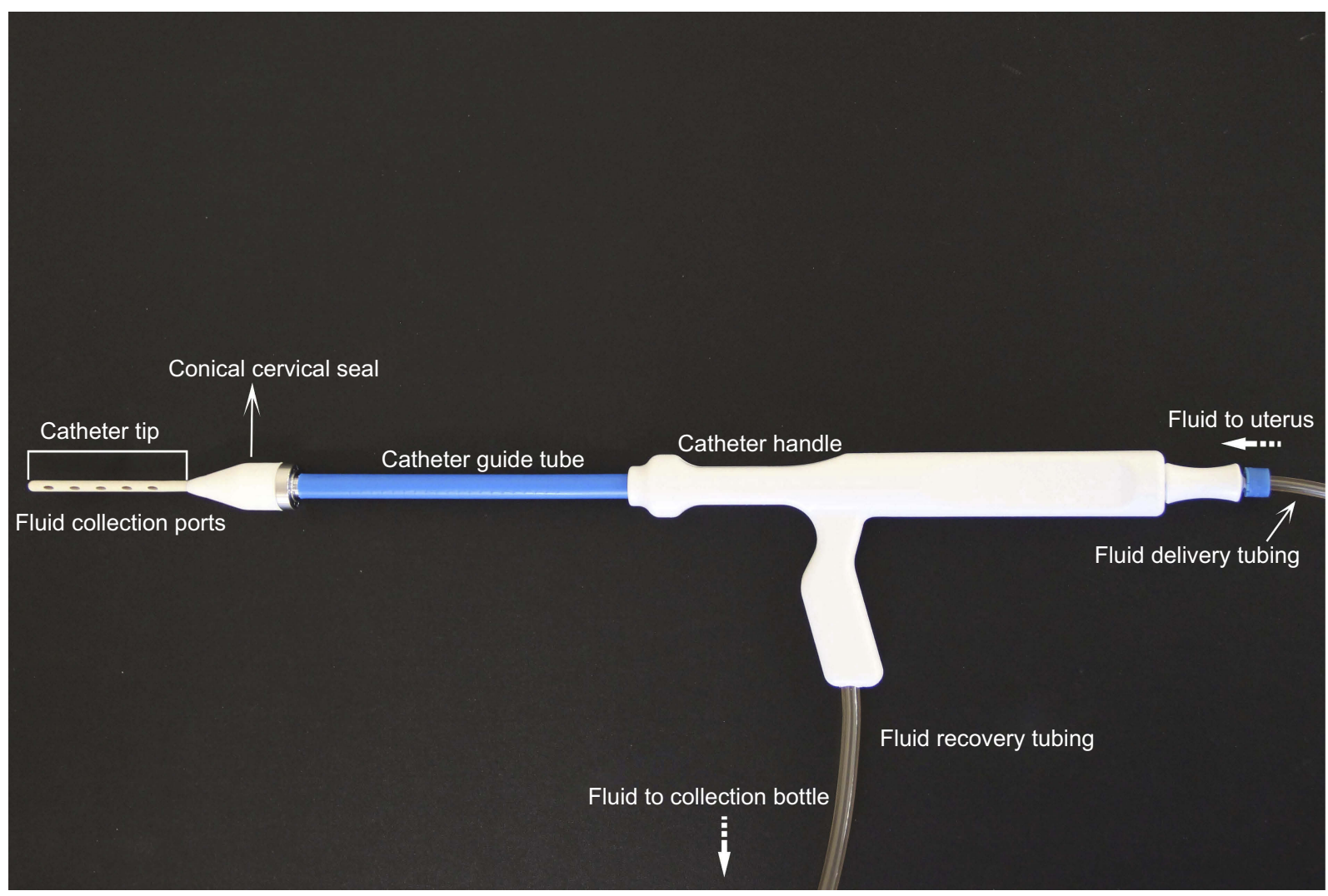

Figure I Current generation catheter. 


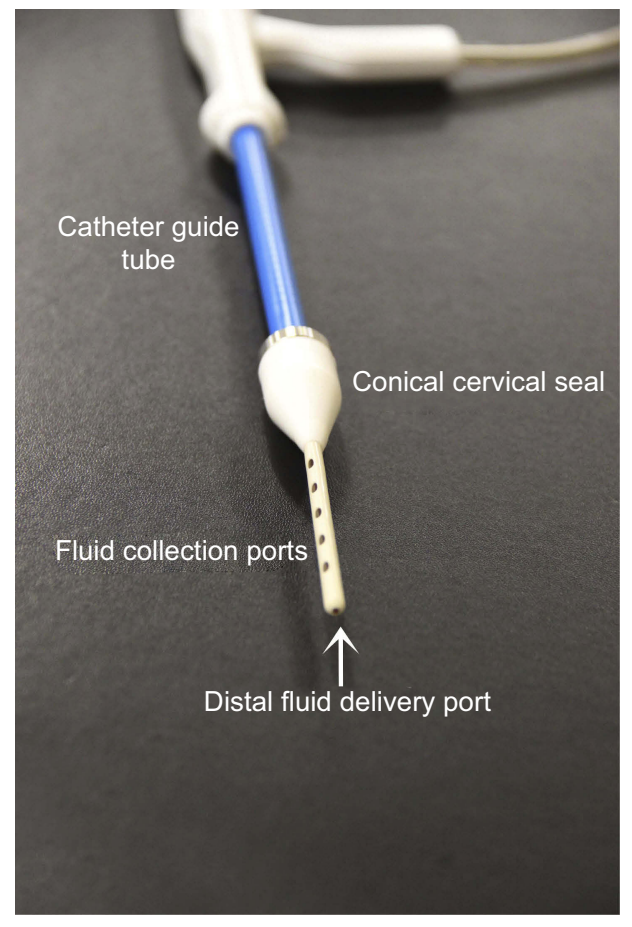

Figure 2 Catheter tip close up.

echogenic and can be shaped to hold memory to traverse different cervical and uterine anatomies.

The catheter tip (Figure 2) contains a single fluid delivery port located at the distal end of the tip. Fluid is delivered through an inner polyamide tube positioned coaxially within the catheter tip. The polyamide tube opening is approximately $0.50 \mathrm{~mm}$ in diameter and egresses from the distal end of the tip. There are ten fluid collection ports configured along the axis of the catheter tip; five collection ports are linearly configured on each side of the catheter tip. This allows for fluid collection to be performed at any location within the uterus as the catheter tip traverses the depth of the uterus. Each collection port is oblong measuring approximately $1.5 \mathrm{~mm} \times 4.5 \mathrm{~mm}$, with a pitch spacing of $10 \mathrm{~mm}$. The oblong shape of the ports is optimal for uterine lavage as it prevents obstruction of the catheter ports with thick cells such as endometrial tissue. Embedded within the catheter tip are two malleable wires with a Brinell Hardness of $\leq \mathrm{b} / 88$. This Brinell Hardness was intended to provide positioning of the catheter tip. At the base of the catheter tip is a conical shaped seal made from compliant silicone. The base of the cone has a $17-\mathrm{mm}$ diameter which tapers down to intimate contact with the catheter tip.

During operation, fluid is delivered from the distal port of the catheter tip directly to the uterine fundus (distal end of the uterus). Fluid hitting the fundus is reflected backwards creating a turbulent lavage action that is distributed throughout the uterus to dislodge ova from hard to reach places.

Ova are thus transported through laminar flow out of the catheter and into the collection bottle. The velocity being sufficiently high such that the fluid inertia will expel the entrained ova out of the endometrium and make them available for collection, the velocity being defined by the following:

$$
V=\frac{r_{0}^{2}-r^{2}}{4 \mu}\left[-\frac{d}{d s}(p+\gamma z)\right]
$$

Of particular interest is the center of the collection tubing where $r=0$, this is where the ova experience the highest inertial forces flowing through the system. Also, of interest are the walls $r^{2}$ where the flow is essentially zero and the ova can stick to the tubing. To mitigate any risk of sticking, a surfactant is applied to the lavage fluid.

\section{Operating protocol}

The uterine lavage process starts weeks prior to the lavage procedure at menses. Subjects begin a daily protocol of fertility drugs to mature multiple follicles in the ovaries. An ovulatory trigger is then administered to release the oocytes from the ovaries into the fallopian tubes. Approximately $35 \mathrm{hrs}$ after the trigger, the patient receives an intrauterine insemination (IUI). The lavage procedure takes place approximately 5 days after IUI.

During the lavage, approximately $100-300 \mathrm{~mL}$ of lavage fluid is delivered into the uterus and collected over a period of between $30 \mathrm{~s}$ and $1 \mathrm{~min}$; this process is managed by the controller. Figure 3 shows a cross-sectional ultrasound view of the uterus intra-lavage with the catheter in its optimal position. The walls of the uterus have been expanded by about $50-70 \mathrm{mmHg}$, and the catheter tip is clearly visible in between the posterior and anterior walls. Uterine lavage cycles with this profile position and uterine expansion have better ova recovery potential than lavages in which the catheter is pressed against the anterior or posterior wall. Ensuring smooth and consistent flow out of the catheter is essential to maintaining the appropriate uterine pressure.

Figure 4 shows a cross-sectional ultrasound view of a uterus intra lavage with the catheter resting against the anterior wall. This is a less than optimal position for the catheter tip as the catheter is buried in the endometrium thus affecting its ability to collect fluid and ova efficiently. Furthermore, the turbulent lavage action will be isolated to the anterior wall thus compromising the catheter's ability to collect ova (oocytes or embryos) along the posterior wall. 


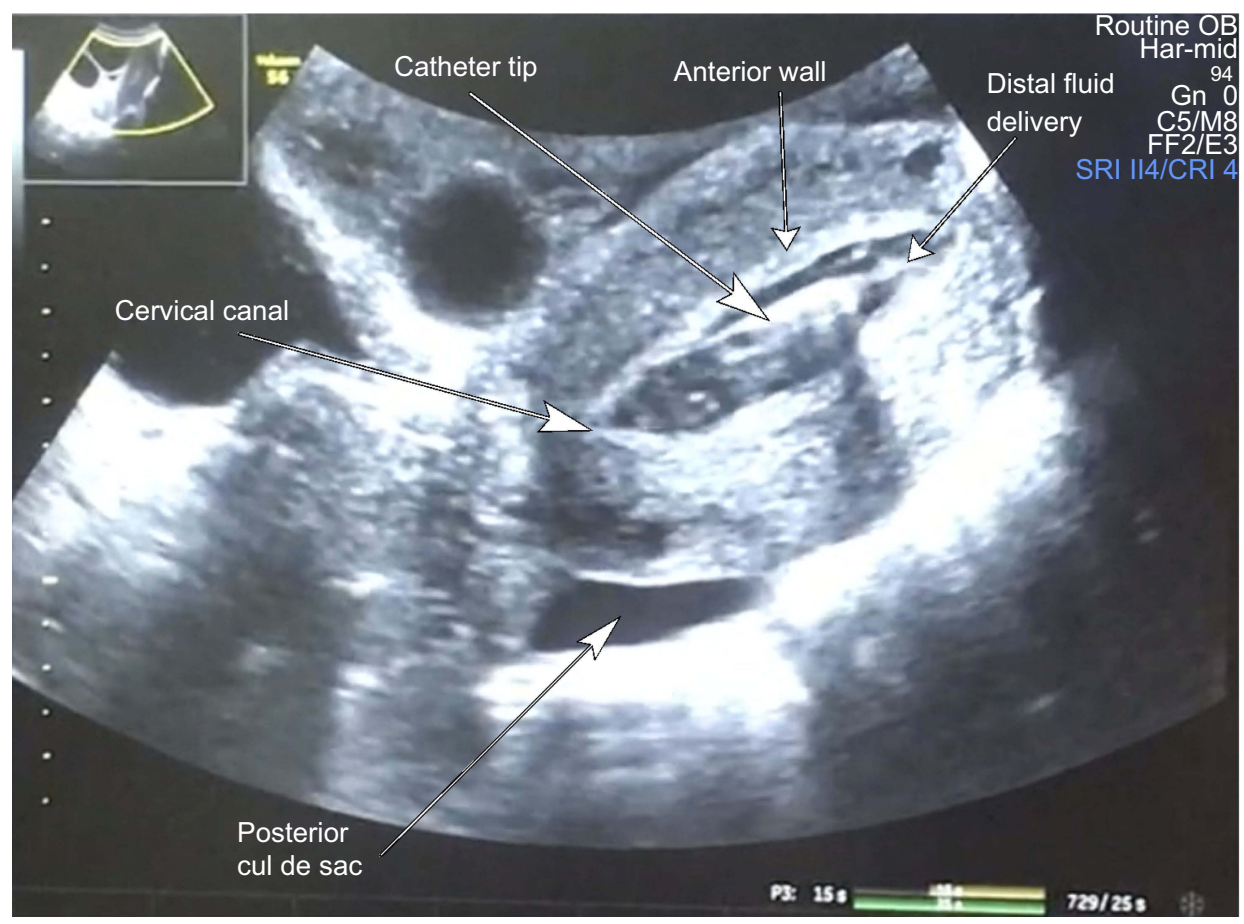

Figure 3 Optimal catheter position intra lavage.

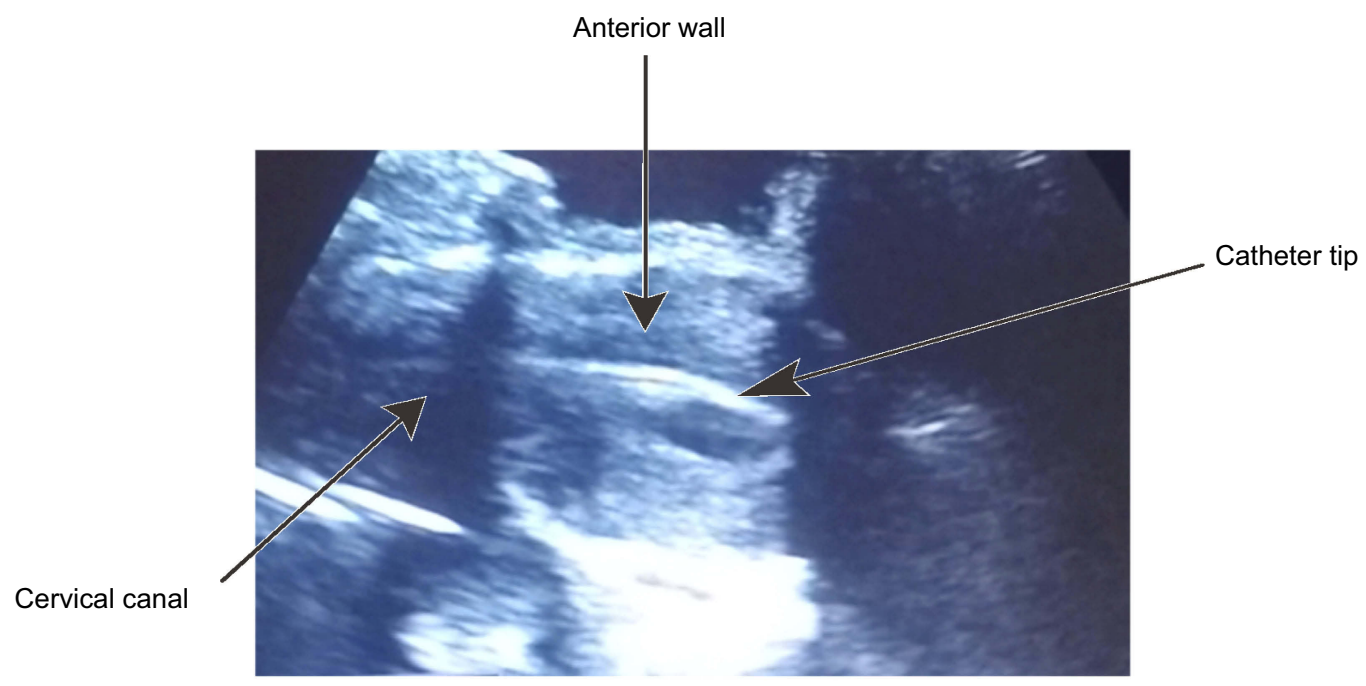

Figure 4 Catheter tip resting against anterior wall intra lavage.

At the end of the procedure, the outflow of fluid is assisted by negative pressure through the system to collect residual fluid in the catheter.

\section{Subject selection}

Subjects enrolled in this study were either oocyte donors or clinical patients recruited by Punta Mita Hospital. Subjects were of reproductive age and screened for normal metabolic health, normal endocrine profiles, normal reproductive anatomy and no infectious diseases. Subjects were allowed to participate in multiple cycles. A total of 81 unique subjects were enrolled in 134 cycles (1.6 cycles/subject). Table 1 details the physical profiles of the subjects. Subjects with a prior history of fertility were preferred as this would mitigate infertility variables that could impact the feasibility results; $86.4 \%$ (70/81) of the subjects were egg donors and $13.6 \%(11 / 81)$ of the subjects were fertility patients seeking to get pregnant. 
Table I Subject characteristics

\begin{tabular}{|l|l|}
\hline Number of stimulated cycles & 134 \\
Number of unique subjects & $8 \mathrm{I}$ \\
Average age & $26.3 \pm 5.3$ \\
Average BMI & $25.6 \pm 4.6$ \\
Obstetrical/gynecologic history & $54.3 \%$ Multiparous (44/8I) \\
& $45.7 \%$ Nulliparous (37/8I) \\
Subject profile & $86.4 \%$ Egg donors (70/8I) \\
& $13.6 \%$ Pregnancy subjects (I I/8I) \\
\hline
\end{tabular}

\section{Results}

\section{Ova recovery}

Uterine lavage procedures were performed once per each stimulation cycle an average of $121 \mathrm{hrs}$ (range 89-144) post IUI. During each procedure, an average of one to three lavage cycles was performed. The catheter tip was able to traverse the cervix in most procedures, but a tenaculum was needed for the majority of lavages (116/118, data not reported in 16/134 cycles) to straighten the cervix for insertion. An average of $109.9 \mathrm{~mL}$ (range $50-170 \mathrm{~mL}$, data not reported in 11 cycles) of lavage media was collected from the lavages. The three lowest collection volumes $(50,50$ and $60 \mathrm{~mL})$ were associated with difficult anatomy.

We define "recovery efficiency" as the number of cycles in which an oocyte or embryo is recovered, divided by the total number of cycles performed. The current recovery efficiency of the device is $53 \%$ (71/134) with a range of $23-80 \%$. It has improved over the course of the study, attributed to optimizations in technique. When looking at embryo recovery efficiency alone, the efficiency is $42 \%(56 / 134)$. To date, eleven cohorts of subjects (134 stimulated cycles) have been completed. Figure 5 shows the recovery efficiency over time of both ova (oocytes and embryos) and embryos alone. The earlier cohorts were associated with the lowest efficiency of $23 \%$ and $31 \%$, respectively. Following the second cohort, the efficiency increased nearly twofold to $54 \%$ and maintained a range of between $40 \%$ and $80 \%$.

In $70 \%(7 / 10)$ of the cohorts, there is a delta between overall ova efficiency and embryo efficiency ranging from $7 \%$ (cohort 7)- $23 \%$ (cohort 6 ). This delta is likely due to biological factors causing some subjects to have improved rates of fertilization over other subjects. The priority of the lavage system is to collect available ova from the uterus, but there is a possibility that all ova collected from a subject may be unfertilized and therefore not clinically useful. Such issues will need to be mitigated by the clinic managing the stimulation cycle to optimize the likelihood the subject will produce fertilized ova.

Proper catheter positioning has been found to contribute significantly to recovery efficiency. In the initial cohorts, the catheter tip was frequently pressed against the endometrial wall as seen in Figure 4. As the catheter is not steerable within the uterus, it was difficult to keep the catheter in the center the endometrium and recovery efficiency was low. With increased practice, the operator was able to manipulate the positioning of the uterus such that the catheter tip was located in the center of the endometrium as shown in Figure 3. This proper

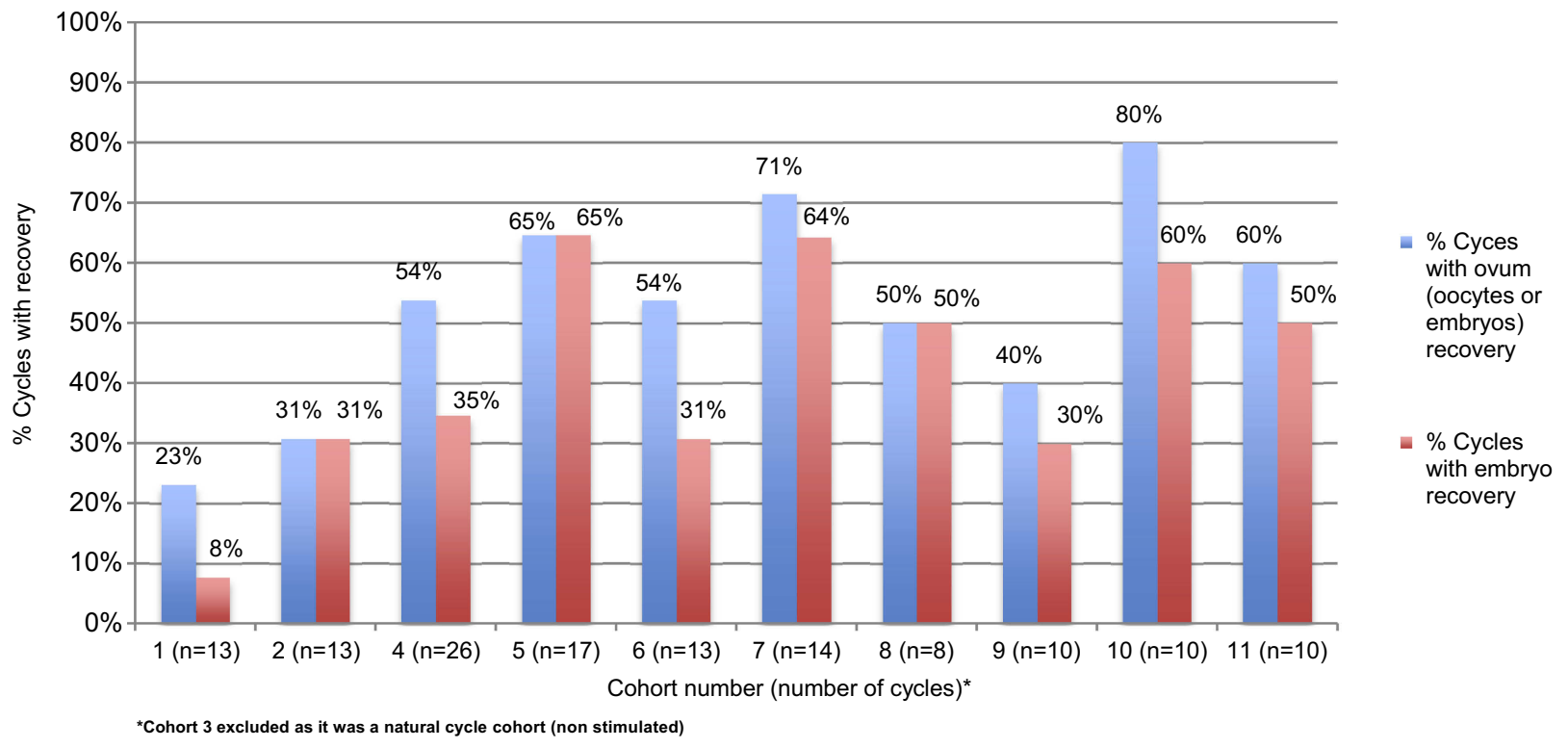

Figure $\mathbf{5}$ Recovery efficiency by the cohort. 
positioning helped maintain the optimal pressure within the uterus and limit over pressurization of the uterine cavity. With proper positioning, it was expected there would be minimal loss of fluid loss out of the fallopian tubes.

A total of 212 ova (oocytes or embryos) were recovered, $36 \%(76 / 212)$ of the ova were unfertilized oocytes, and $64 \%$ (136/212) were embryos. On average the lavage device recovered 1.6 ova/lavage (212 ova/134 lavages, range $0-17$ ova). The unfertilized oocytes serve as markers of efficiency but serve no clinical utility as they are beyond the window of fertilization, oocytes can only be fertilized within $24 \mathrm{hrs}$ of ovulation.

Blastocysts collected were graded per the Gardner Scale of Morphology which enables an evaluation of the quality of the blastocysts. ${ }^{1}$ Gradings were assigned by the Punta Mita embryologist and categorized as "good morphology" or "poor morphology". Of the 96 blastocysts, 95/96 were graded and $73.7 \%$ (70/95) were of good morphology, $26.3 \%(25 / 95)$ were graded as poor quality.

\section{Non-recovery cycles}

As previously stated, we collected ova in $53 \%$ of uterine lavage cycles. Thus, about half of the cycles result in no ova recovery.

It is difficult to know whether ova are present in the uterus at the time of lavage, and we expect there will be cycles where there are no ova to collect. Through natural conception (non-stimulated), there is a $10-20 \%$ chance of pregnancy/month, we can expect an embryo to be present in at least $10-20 \%$ of natural cycles. Administering gonadotropins may increase this probability. For those $<35$ years undergoing Gonadotropin/IUI, the chance of pregnancy is approximately $33 \%$ and for those $>35$ years this number drops to $15 \%{ }^{2}$ Thus, in approximately $15-33 \%$ of uterine lavage cycles (age dependent), there will be at least one embryo to collect. This estimate, however, does not account for embryos which were present but failed to implant. Embryos have an approximately 50\% chance of pregnancy, thus we estimate that embryos may be present in $30-66 \%$ of uterine lavage cycles. ${ }^{3}$

In cycles where there is zero collection, there may be nothing to collect, or the device may have been unable to collect ova present in the uterus. The following is a list of known reasons for why zero recovery cycles may occur.

- Ovulation failure: no ova in the uterus ${ }^{4,5}$

- Tubal obstruction: blocked tubal transport ${ }^{6}$
- Highly viscous endometrial fluid: entrapped ova in mucus $^{7}$

- Early implantation: ova implant prior to lavage ${ }^{8}$

- Incorrect procedure timing: ova have either left, or not arrived in the uterus ${ }^{9}$

\section{Safety}

No serious adverse events were reported during the procedures or at follow-up visits. Subjects reported events such as bleeding, cramping, abdominal pain, nausea, headache and lightheadedness post lavage, all of which are symptoms commonly associated with intrauterine procedures. Symptoms subsided within days from the lavage procedure and were mild in nature. Subjects were followed up with for 30 days post-lavage for confirmation of safety.

Immediately after lavage, subjects were given a GnRH antagonist to accelerate menses and minimize embryo retention. Eight days after the lavage, subjects were tested for pregnancy via human chorionic gonadotropin (hCG). At this visit, $8 \%(11 / 134)$ of subjects had a positive hCG result, an adverse event, with $63 \%(7 / 11)$ of the positive hCGs resulting from the same cohort (Cohort 5, Figure 5). Subjects with elevated hCG levels were monitored until such time the hCG levels were undetectable. After Cohort 5 , the antagonist dosage was changed and only three subsequent positive hCGs were observed in the remaining 65 cycles. The subjects with elevated hCG levels were asymptomatic, and no visible signs of an intrauterine or ectopic pregnancy were observed. The positive hCGs either spontaneously resolved $(\mathrm{n}=2 / 11)$, resolved after endometrial curettage $(n=2 / 11)$, resolved after Methotrexate $(n=3 / 11)$, or resolved after both endometrial curettage and Methotrexate $(n=4 / 11)$.

\section{Discussion}

In vitro culture can cause genetic mutations to embryos, and aneuploidy rates are known to vary by clinic; some clinics produce more aneuploid IVF embryos and this is likely the result of variable embryo handling practices and lab conditions. ${ }^{10-12}$ This may explain why children born from IVF are more likely than naturally conceived infants to have multiple major defects (eg, cardiovascular, urogenital, chromosomal and musculoskeletal defects), lower birth weights, and chromosomal alterations (eg, fetal sex chromosomal anomalies, de novo anomalies, Y-chromosome microdeletions) when compared to naturally conceived children. ${ }^{13,14}$ Uterine lavage can bypass these known issues by providing a platform to retrieve in vivo embryos from the 
uterus. These embryos are less likely to be affected by issues associated with in vitro culture and lab conditions as embryogenesis is completed within the body. After lavage, embryos are ready to be biopsied, cryopreserved or transferred (Table 2).

We have shown that uterine lavage may be a viable method for retrieving in vivo embryos. The recovered embryos are of good quality and not damaged by the lavage procedure. The majority of embryos recovered were blastocysts thus minimizing in vitro culture. By minimizing in vitro culture, many risks to the embryos are mitigated and there may be a significant cost saving to the patient as they will not have to pay for evaluation of the embryos for 5-6 days. Furthermore, uterine lavage can expand access to genetic diagnostic capabilities to fertile couples who do not have to undergo IVF to screen their embryos.

In the US in 2010, there were a total of 332,962 aneuploid pregnancies ( $\mathrm{J}$ Rosen, Women and Infants Hospital of Rhode Island, Personal communication, June 2012). Many of these aneuploid pregnancies will result in a spontaneous abortion, of which $50-80 \%$ of such abortions are due to aneuploidy. ${ }^{15}$ Enabling uterine lavage to screen in vivo embryos prior to pregnancy could significantly reduce the number of aneuploid pregnancies and reduce the incidence of spontaneous abortions. Further to this, there are a significant number of pregnancies affected by single gene mutations that can also be identified by uterine lavage + preimplantation genetic testing. Among just $15 / 6500$ most common single gene mutations, there are an estimated 20,000 births of children with disease in the USA alone per year ( $\mathrm{J}$ Rosen, Women and Infants Hospital of Rhode Island, Personal communication, June 2012). An ART procedure, such as uterine lavage, may be an acceptable treatment option to expand patient access to PGT, therefore assisting in the potential reduction and incidence of genetic disease. ${ }^{16}$

The lavage system is still undergoing improvements. Further improvements will be implemented to facilitate the procedure technique such that the operator does not have difficulty in keeping the catheter tip centered. As the catheter is not fully efficient, the risk of positive hCG post lavage needs to be weighed in the risk/benefit profile. A GhRH antagonist injection is given to each patient after the lavage procedure to induce menses such that non-recovered embryos cannot implant. Thus far the GnRH antagonist has been effective. The positive hCG rate post lavage is approximately $8 \%$ (11/134), with $63 \%(7 / 11)$ of those positive hCGs coming from cohort 5. We hypothesize there were causal factors associated with the high incidence of positive hCG of that cohort that is independent of the lavage procedure. Excluding that cohort, the overall rate of positive $\mathrm{hCG}$ is $3.4 \%$ (4/ 117). Further studies will be conducted to understand the risk of positive hCG post lavage, but it is expected that with a more efficient device, the rate of positive hCG will decrease further improving the risk/benefit profile of the lavage system.

\section{Conclusion}

The Previvo Uterine Lavage System is an innovative medical device that can effectively and safely recover highquality in vivo embryos from a woman's uterus.

This body of testing is the most significant progress made in three decades toward advancing uterine lavage as a treatment option for assisted reproduction. Device upgrades are currently being implemented to the catheter and anticipated to significantly increase current efficiencies and further validate the results of this work. Further work

Table 2 IVF and uterine lavage procedure attributes

\begin{tabular}{|l|l|l|}
\hline IVF procedure attribute & IVF & Uterine lavage \\
\hline $\begin{array}{l}\text { Superovulation with fertility drugs } \\
\text { Aspiration of oocytes (unfertilized eggs) from the } \\
\text { ovaries }\end{array}$ & $\begin{array}{l}\text { Required } \\
\text { Required - needle inserted transvaginally } \\
\text { In vitro fertilization (external fertilization of eggs) }\end{array}$ & $\begin{array}{l}\text { Optional - natural cycles can be performed } \\
\text { Ret required }\end{array}$ \\
In vitro culture & Required & Not required - natural fertilization \\
& Optional & $\begin{array}{l}\text { Not required - naturally fertilized embryos } \\
\text { recovered at lavage after in vivo culture }\end{array}$ \\
Embryo biopsy & Optional & Optional \\
Embryo cryopreservation & Optional & Optional \\
Preimplantation genetic testing (PGT) & Optional & Optional \\
Embryo transfer & & \\
\hline
\end{tabular}


will be performed to understand the implantation potential of these in vivo embryos.

Successful implementation of uterine lavage has positive implications for both patients and assisted reproductive technology in general. In-vivo embryos may reveal critical information about the earliest phases of embryo development, which has medical and technological implications well beyond the scope of this work.

\section{Ethics approval}

The work described in this paper was performed at the Punta Mita Hospital in Punta Mita, Mexico. The study received Institutional Review Board (IRB) approval from Western IRB (Puyallup, WA, USA) and local Mexican government approval from Servicios de Salud de Nayarit (Tepic, Mexico). The study is currently registered on clinicaltrials.gov (NCT03426007) and has been performed in accordance with the Declaration of Helsinki.

\section{Informed consent}

Written informed consent was received from all subjects in which subjects acknowledged the benefits/risks of the study and agreed to the publication of de-identified study data gathered through their participation.

\section{Data sharing statement}

The authors do not intend to share any further de-identified participant data.

\section{Acknowledgment}

All funding provided by Previvo Genetics, Inc.

\section{Disclosure}

Alexander Nadal declares he is a salaried employee of Previvo Genetics, and holds stock options in Previvo Genetics. Dr. Sam Najmabadi declares that he holds shares of Previvo Genetics, and was provided funding to perform this study by Previvo Genetics. Bruce Addis declares he is a salaried employee of Previvo Genetics, holds stock options in Previvo Genetics, and is a named co-inventor on a provisional patent application with rights assigned to Previvo Genetics. Dr. John E Buster reports he is a salaried employee of Previvo Genetics, is a member on the Previvo Genetics Board of Directors and Scientific Advisory Board, and holds shares in Previvo Genetics. In addition,
Dr. Buster is a named co-inventor on multiple granted patents and patent applications, with all rights assigned to Previvo Genetics. All authors report no other conflicts of interest in this work.

\section{References}

1. Gardner DK, Lane M, Stevens J, Schlenker T, Schoolcraft WB. Blastocyst score affects implantation and pregnancy outcome: towards a single blastocyst transfer. Fertil Steril. 2000;73(6):1155-1158.

2. Guzick DS, Overstreet JW, Hill JA, Vogel DL. Efficacy of superovulation and intrauterine insemination in the treatment of infertility. N Engl J Med. 1999;340(3):177-183.

3. Gardner DK, Vella P, Lane M, Wagley L, Schlenker T, Schoolcraft WB. Culture and transfer of human blastocysts increases implantation rates and reduces the need for multiple embryo transfers. Fertil Steril. 1998;69(1):84-88.

4. Stanger JD, Yovich JL. Failure of human oocyte release at ovulation. Fertil Steril. 1984;41(6):827-832. doi:10.1016/S0015-0282(16) 47893-3

5. Qublan H, Amarin Z, Nawasreh M, et al. Luteinized unruptured follicle syndrome: incidence and recurrence rate in infertile women with unexplained infertility undergoing intrauterine insemination. Hum Reprod. 2006;21(8):2110-2113. doi:10.1093/humrep/del113

6. Kovacs GT. The Subfertility Handbook: A Clinician's Guide. Cambridge UK: Cambridge University Press; 1997.

7. Saadat P, Boostanfar R, Slater CC, Tourgeman DE, Stanczyk FZ, Paulson RJ. Accelerated endometrial maturation in the luteal phase of cycles utilizing controlled ovarian hyperstimulation: impact of gonadotropin-releasing hormone agonists versus antagonists. Fertil Steril. 2004;82(1):167-171. doi:10.1016/j. fertnstert.2003.11.050

8. Kirkegaard K, Sundvall L, Erlandsen M, Hindkjær JJ, Knudsen UB, Ingerslev HJ. Timing of human preimplantation embryonic development is confounded by embryo origin. Hum Reprod. 2015;31 (2):324-331. doi:10.1093/humrep/dev296

9. Croxatto HB. Physiology of gamete and embryo transport through the Fallopian tube. Reprod Biomed Online. 2002;4(2):160-169. doi:10.1016/S1472-6483(10)61935-9

10. Munné S, Alikani M, Ribustello L, Colls P, Martínez-Ortiz PA, McCulloh DH. Euploidy rates in donor egg cycles significantly differ between fertility centers. Hum Reprod. 2017;32(4):743-749. doi:10.1093/humrep/dex031

11. Munne S, Magli C, Adler A, et al. Treatment-related chromosome abnormalities in human embryos. Hum Reprod. 1997;12(4):780-784. doi:10.1093/humrep/12.4.780

12. Munné S, Alikani M. Culture-induced chromosome abnormalities: the canary in the mine. Reprod Biomed Online. 2011;22(6):506-508. doi:10.1016/j.rbmo.2011.02.011

13. Hansen M, Kurinczuk JJ, Bower C, Webb S. The risk of major birth defects after intracytoplasmic sperm injection and in vitro fertilization. N Engl J Med. 2002;346(10):725-730. doi:10.1056/NEJMoa010035

14. Kurinczuk JJ. Safety issues in assisted reproduction technology: From theory to reality - just what are the data telling us about ICSI offspring health and future fertility and should we be concerned? Hum Reprod. 2003;18(5):925-931. doi:10.1093/humrep/deg217

15. Simpson JL. Causes of fetal wastage. Clin Obstet Gynecol. 2007;50 (1):10-30. doi:10.1097/GRF.0b013e31802f11f6

16. Buster JE, Carson SA. Genetic diagnosis of the preimplantation embryo. Am J Med Genet. 1989;34(2):211-216. doi:10.1002/ ajmg. 1320340216 


\section{Publish your work in this journal}

Medical Devices: Evidence and Research is an international, peerreviewed, open access journal that focuses on the evidence, technology, research, and expert opinion supporting the use and application of medical devices in the diagnosis, monitoring, treatment and management of clinical conditions and physiological processes. The identification of novel devices and optimal use of existing devices which will lead to improved clinical outcomes and more effective patient management and safety is a key feature of the journal. The manuscript management system is completely online and includes a very quick and fair peer-review system. Visit http:// www.dovepress.com/testimonials.php to read real quotes from published authors.

Submit your manuscript here: https://www.dovepress.com/medical-devices-evidence-and-research-journal 